\title{
The True Surface Mass density of Cold Dark Matter Halos
}

\author{
Janne Holopainen ${ }^{1} \dagger$, E. Zackrisson ${ }^{1,2,3}$, A. Knebe ${ }^{4}$, P. Nurmi ${ }^{1}$, \\ P. Heinämaki ${ }^{1}$, S. Gill ${ }^{5}$, C. Flynn ${ }^{1}$ and T. Reihm ${ }^{2}$ \\ ${ }^{1}$ Tuorla Observatory, University of Turku, Väisäläntie 20, Piikkiö, FI-21500, Finland \\ ${ }^{2}$ Stockholm Observatory, AlbaNova University Center, 10691 Stockholm, Sweden \\ ${ }^{3}$ Department of Astronomy and Space Physics, Box 515, SE-75120 Uppsala, Sweden \\ ${ }^{4}$ Astrophysikalisches Institut Potsdam, An der Sternwarte 16, 14482 Potsdam, Germany \\ ${ }^{5}$ Department of Astronomy, Columbia University, 550 West 120th Street, New York, NY \\ 10027, USA
}

\begin{abstract}
The cold dark matter (CDM) scenario generically predicts the existence of triaxial dark matter halos which contain notable amounts of substructure. However, analytical halo models with smooth, spherically symmetric density profiles are routinely adopted in the modelling of light propagation effects through such objects. In this paper, we report the biases introduced by this procedure by comparing the surface mass densities of actual N-body halos against the widely used analytical model suggested by Navarro, Frenk and White (1996) (NFW). We conduct our analysis in the redshift range of $0.0-1.5$.

In cluster sized halos, we find that triaxiality can cause scatter in the surface mass density of the halos up to $\sigma_{+}=+60 \%$ and $\sigma_{-}=-70 \%$, where the 1- $\sigma$ limits are relative to the analytical NFW model given value. Subhalos can increase this scatter to $\sigma_{+}=+70 \%$ and $\sigma_{-}=-80 \%$. In galaxy sized halos, the triaxial scatter can be as high as $\sigma_{+}=+80 \%$ and $\sigma_{-}=-70 \%$, and with subhalos the values can change to $\sigma_{+}=+40 \%$ and $\sigma_{-}=-80 \%$.

We have developed an analytical model for the surface mass density scatter as a function of distance to the halo centre, halo redshift and halo mass. The analytical description enables one to investigate the reliability of results obtained with simplified halo models. Additionally, it provides the means to add simulated surface density scatter to analytical density profiles. We have tested our model on the calculation of microlensing optical depths for MACHOs in CDM halos.
\end{abstract}

Keywords. methods: n-body simulations, dark matter, galaxies: structure, galaxies: halos, gravitational lensing

\section{Introduction}

The light emitted from high-redshift objects such as quasars, supernovae, gamma-ray bursts, galaxies and galaxy clusters will typically have to pass through many dark matter halos before reaching an observer on Earth. Several investigations have already indicated that smooth and/or spherical halo models may lead to incorrect results when treating the gravitational lensing effects associated with such foreground mass condensations (e.g. Bartelmann \& Weiss 1994; Holopainen et al. 2006).

There are several situations in gravitational lensing when realistic estimates of the surface mass density along a given line of sight through a dark halo may be important. Examples include the calculation of image separations in strong lensing by subhalos located in the external potential of its host halo (Oguri 2005), attempts to correct the luminosities of supernovae type Ia for the magnification by foreground halos (e.g. Gunnarsson

\section{† email: jaolho@utu.fi}


2004) and estimates of the distribution of microlensing optical depths for high-redshift MACHOs (e.g. Zackrisson \& Riehm 2007). Other applications include the assessments of light propagation effects in models with non-zero coupling betweeen dark matter particles and photons (e.g. Profumo \& Sigurdson 2007).

\section{Results}

We have compared a sample of CDM N-body halos to the smooth, spherically symmetric NFW density profile model in three dimensions. The differences in surface mass density of the halos and the model are studied, and an analytical description of the differences is constructed. This description can be used to estimate or reproduce the differences between CDM N-body halos and, in principle, any analytical halo model. It can be used in applications in which the line-of-sight surface mass densities of CDM halos play an important role (e.g microlensing).

Our halo sample consists of 27 independent CDM halos at $\sim 10$ redshift snapshots between $0.0<z<1.5$. The halos are extracted from three cosmological simulations with comoving box sizes of $10 h^{-1} \mathrm{Mpc}, 40 h^{-1} \mathrm{Mpc}$ and $64 h^{-1} \mathrm{Mpc}$. The halos are treated both with and without their subhalos, and the halo sample is divided in two mass classes, separated by a mass gap at $M \sim 10^{13} \mathrm{M}_{\odot} h^{-1}$. The analytical description is given for all four cases.

We find that the surface mass density of the halos can deviate from the spherical model considerably. At minimum, with zero impact parameter and redshift, the 1- $\sigma$ limits around the NFW surface mass density are close to $\sigma= \pm 20 \%$ or $\sigma= \pm 30 \%$, depending which halos are under investigation. At maximum, with impact parameter close to $r_{\text {vir }}$ and $\mathrm{z}=1.5$, the values can be as high as $\sigma_{+}=+70 \%$ and $\sigma_{-}=-80 \%$. The geometric mean of the surface mass density is offset from the NFW predicted value by $-3 \%$ to $-44 \%$, depending on the case.

We also find that the departure from the NFW profile is log-normally distributed around the model value. In most cases, the median of the surface mass density of the halos is slightly lower than predicted by the NFW profile. The variation of the surface mass density around the NFW value grows with increasing impact parameter and redshift.

As an application, we implemented our analytical description to the optical depth calculations of MACHOs. In this case, we find that the variance in surface mass density due to halo shapes can be overwhelmed by the variance caused by random impact parameters between halos on the same sightline.

The results of this proceedings paper are presented in much more detail in another paper submitted to MNRAS by the same authors and expected to be published before the end of 2007.

\section{References}

Bartelmann, M. \& Weiss, A. 2004, A\&A, 287, 1

Gunnarsson, C. 2004, JCAP 03, 2

Holopainen, J., Flynn, C., Knebe, A., Gill, S. P. \& Gibson, B. K. 2006, MNRAS 368, 1209

Navarro, J. F., Frenk, C. S. \& White, S. D. M. 1996, ApJ, 462, 563

Oguri, M. 2005, MNRAS 367, 1241

Profumo, S. \& Sigurdson, K. 2007, Phys.Rev. D75, 023521

Zackrisson, E. \& Riehm, T. 2007, A\&A, submitted 